\title{
Crescimento e desenvolvimento puberal na adolescência
}

\section{Growth and puberal development in adolescence}

\author{
Benito Lourenço', Lígia Bruni Queiroz ${ }^{2}$
}

\begin{abstract}
Lourenço B, Queiroz LB. Crescimento e desenvolvimento puberal na adolescência. Rev Med (São Paulo). 2010 abr.-jun.;89(2):70-5.

RESUMO: A puberdade é um período da adolescência que se caracteriza pelas mudanças biológicas que ocorrem nessa fase da vida. Com duração de cerca de dois a quatro anos, ela é marcada por crescimento esquelético linear; alteração da forma e composição corporal; desenvolvimento de órgãos e sistemas e desenvolvimento de gônadas e caracteres sexuais secundários. Este artigo se propõe a discorrer sobre esses eventos que compõem a puberdade, bem como os fatores que podem influenciá-los.
\end{abstract}

DESCRITORES: Puberdade; Adolescentes; Crescimento e desenvolvimento.

\begin{abstract}
A adolescência compreende a faixa etária situada entre os dez e vinte anos incompletos, segundo a Organização Mundial de Saúde, e se constitui como uma fase crítica do processo de crescimento e desenvolvimento humano, marcada por numerosas transformações relacionadas aos aspectos físicos, psíquicos e sociais do indivíduo ${ }^{8}$. A puberdade é caracterizada pelas mudanças biológicas que se manifestam na adolescência, e representam, para o ser humano, o início da capacidade reprodutiva. A puberdade não é, portanto, sinônimo de adolescência, mas uma parte dela ${ }^{8}$. Constitui-se por um período relativamente curto, de cerca de dois a quatro anos de duração, no qual ocorrem todas as modificações físicas desse momento de transição da
\end{abstract}

infância para a idade adulta. Essas transformações somáticas que ocorrem na adolescência têm caráter universal, ou seja, representam um fenômeno comum a todos os indivíduos nessa fase da vida. Embora ainda persistam dúvidas sobre a complexa dinâmica da ativação puberal, sabe-se que esse momento se inicia apos a reativação de neurônios hipotalâmicos, que secretam, de uma maneira pulsátil bastante específica, o hormônio liberador de gonadotrofinas $(\mathrm{GnRH})$. A secreção desse resulta na consequente liberação também pulsátil dos hormônios luteinizante (LH) e folículo-estimulante (FSH) pela glândula hipófise. Isso ocorre inicialmente durante o sono e, mais tarde, estabelece-se em ciclo circadiano ${ }^{2}$.

O crescimento e o desenvolvimento são

\footnotetext{
${ }^{1}$ Médico Assistente da Unidade de Adolescentes do Instituto da Criança do HC-FMUSP. Médico Assistente da Clínica de Adolescência do Departamento de Pediatria da Faculdade de Ciências Médicas da Santa Casa de São Paulo.

${ }^{2}$ Médico Assistente da Unidade de Adolescentes do Instituto da Criança do HC-FMUSP.

Endereço para correspondência: Benito Lourenço. Rua Dr. Enéas de Carvalho Aguiar, 647 - São Paulo, SP, CEP 05403-000. e-mail: benitol@uol.com.br
} 
eventos geneticamente programados, da concepção ao amadurecimento completo, porém fatores inerentes ao próprio indivíduo (constitucionais ou intrínsecos), e outros, representados por circunstâncias ambientais, podem induzir modificações nesse processo. Fatores climáticos, socioeconômicos, hormonais, psicossociais e, sobretudo, nutricionais são alguns dos interferentes do processo de crescimento e desenvolvimento ${ }^{7}$.

O desenvolvimento dos caracteres sexuais é mais tardio nas classes de menor nível socioeconômico ${ }^{4,12}$. A nítida interferência de fatores extrínsecos (ambientais) na maturação puberal é claramente observada, por exemplo, quando se estuda o fenômeno menarca, que é a primeira menstruação da menina adolescente. A menarca, além de ser um indicador de maturação biológica, também mostra as mudanças que ocorrem com o desenvolvimento social e econômico das populações ${ }^{12}$. As meninas de "status" socioeconômico mais elevado apresentam a primeira menstruação mais precocemente do que aquelas menos favorecidas, mesmo residentes no mesmo país ${ }^{9}$. No Brasil, esse fenômeno também se observa nas classes sociais mais favorecidas e provavelmente devido às melhorias das condições nutricionais e estímulos emocionais. A importância prática, por consequência, envolve a seguinte concepção: quanto mais precoce ocorrer, mais exposta estará a adolescente à gestação ${ }^{12}$. O desenvolvimento puberal mais precoce estaria relacionado à influência positiva da melhoria das condições de vida e principalmente do estado nutricional ${ }^{7}$.

Uma característica própria da puberdade é a sua variabilidade. A idade cronológica não se constitui como um bom indicador para a avaliação de adolescentes. É comum que adolescentes de diferentes grupos etários encontrem-se no mesmo estágio de desenvolvimento. Daí, a necessidade da utilização de critérios de maturidade fisiológica para o acompanhamento do desenvolvimento puberal ${ }^{4}$.

De forma didática, considera-se que a puberdade é caracterizada, fundamentalmente, pelos seguintes eventos: corporal;

- Crescimento esquelético linear;

- Alteração da forma e composição

- Desenvolvimento dos órgãos e sistemas;

- Desenvolvimento das gônadas e dos caracteres sexuais secundárias.

\section{Crescimento esquelético}

$\mathrm{Na}$ adolescência, observa-se um intenso crescimento do esqueleto, denominado estirão puberal. Para a compreensão de tal fenômeno, faz-se necessária a análise da curva de velocidade de crescimento (Figura 1), na qual os ganhos de altura no tempo são projetados em função da idade, representada pela unidade de centímetros por ano (cm/ano). Nessa fase da vida, o adolescente apresenta grande aquisição pôndero-estatural, ganhando cerca de $50 \%$ do seu peso adulto e $20 \%$ de sua estatura final ${ }^{11}$.

O crescimento esquelético ocorre de forma não-linear, com velocidades variáveis de acordo com a fase da vida considerada, e suscetíveis a influências externas como: oferta alimentar, aspectos psicossociais e ambientais, bem como à ação hormonal predominante em cada fase ${ }^{11}$.

O crescimento intrauterino é composto por uma fase inicial (embrionária) com intensa proliferação celular, na qual o incremento somático chega a valores da ordem de $10 \mathrm{~cm}$ por mês (entre o quarto e quinto meses de gestação). Esse período inicial é sucedido por uma fase de menor crescimento estatural, embora marcado por um maior incremento de peso fetal. Considera-se, portanto, que a aceleração do crescimento intrauterino ocorre particularmente na primeira metade da gestação, havendo uma desaceleração no final do período gestacional ${ }^{2}$. Ao nascer, o ser humano apresenta elevada velocidade de crescimento, porém já em desaceleração, que pode ser graficamente observado na Figura 1.

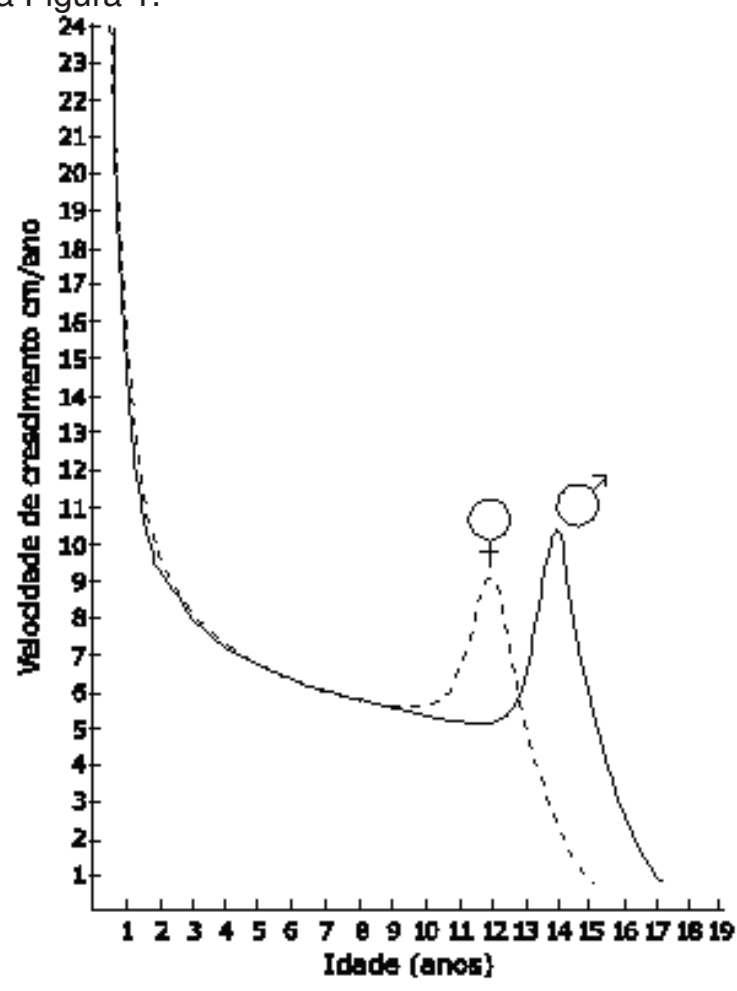

FIGURA 1: Curva de velocidade de crescimento expressa pela idade (VC) 
Lourenço B, Queiroz LB. Crescimento de desenvolvimento puberal na adolescência.

A observação da curva de velocidade de crescimento permite a identificação de três momentos fundamentais do crescimento humano:

Fase 1 (lactância): fase de crescimento rápido, porém desacelerado. A velocidade de crescimento do primeiro ano de vida é a mais alta da vida extrauterina e é cerca de $25 \mathrm{~cm} / \mathrm{ano}$, reduzindose drasticamente nos dois primeiros anos de vida;

Fase 2 (infância propriamente dita): fase de crescimento lento, mais estável e constante. São comuns nos consultórios pediátricos queixas familiares do tipo "meu filho não cresce", por ser um momento de baixa velocidade de crescimento, particularmente quando comparada à fase pregressa. A velocidade média varia de 4 a $6 \mathrm{~cm} / a n o$ e é chamada infantil ou pré-puberal, pois somente se modifica na fase seguinte;

Fase 3 (puberdade): novamente uma fase de crescimento rápido, com aceleração e posterior desaceleração, até, finalmente, o término do processo de crescimento.

Período de intenso crescimento esquelético, a adolescência é considerada como uma fase de grande vulnerabilidade ao desenvolvimento humano, cuja suscetibilidade aos agravos externos pode ocasionar prejuízos irreparáveis à estatura final do indivíduo. Portanto, o adolescente merece um enfoque preventivo de saúde e uma atenção diagnóstica especial em relação à apresentação de doenças crônicas, transtornos alimentares e distúrbios nutricionais ${ }^{6}$.

O estirão de crescimento ou estirão puberal é composto, portanto, pelo período de aceleração da velocidade de crescimento (VC), até atingir um valor de pico (PVC), e subsequente desaceleração até o término do crescimento. O PVC no sexo masculino pode chegar a valores de 10-12 cm/ano e, no sexo feminino, de 8-10 cm/ano $6,8,11$.

O estirão puberal não ocorre de maneira uniforme. Inicia-se pelos membros, seguindo uma direção distal-proximal, ou seja, pés e mãos, inicialmente, seguindo-se pernas e membros superiores, conferindo ao corpo do adolescente um aspecto desarmônico ou de desproporcionalidade, que volta a ser harmônico e proporcional ao término do estirão, após o crescimento do tronco, principal responsável pela estatura final do indivíduo ${ }^{8}$. corporal

Alteração da forma e da composição

Na puberdade, são estabelecidas as distintas formas corporais masculinas e femininas, fenômeno denominado dimorfismo sexual, resultante do desenvolvimento esquelético, muscular e do tecido adiposo $^{1,8}$. A forma do corpo de um bebê ou de uma criança não permite a diferenciação masculino/ feminino, aspecto que se torna nítido durante a puberdade. O depósito de gordura nas meninas ocorre principalmente na região das mamas e dos quadris, e confere um aspecto característico do corpo feminino. Nos homens, o crescimento do diâmetro biacromial (entre ombros), conferindo relação biacromial/bi-ilíaco elevada, associado ao desenvolvimento muscular na região da cintura escapular, define a forma masculina ${ }^{1}$.

A composição corporal do adolescente oscila em função da maturação sexual. A idade da menarca representa o inicio da desaceleração do crescimento que ocorre no final do estirão puberal, e o maior acúmulo de tecido adiposo. Para os meninos, o pico de crescimento coincide com a fase adiantada do desenvolvimento dos genitais e pilosidade pubiana, momento em que também ocorre desenvolvimento acentuado de massa magra e muscular ${ }^{1,3,7}$. sistemas

\section{Desenvolvimento de todos os órgãos e}

Com exceção do tecido linfoide, que apresenta involução progressiva a partir da adolescência, e do tecido nervoso (praticamente com todo o seu crescimento já estabelecido), todos os órgãos e sistemas se desenvolvem durante a puberdade, sobretudo os sistemas cardiocirculatório e respiratório ${ }^{8}$. O aumento da capacidade física observado na puberdade é mais marcante no sexo masculino, e é resultante do desenvolvimento do sistema cardiorrespiratório, das alterações hematológicas (aumento da eritropoiese) e do aumento da massa muscular, da força e da resistência física ${ }^{8}$.

Desenvolvimento gonadal e das características sexuais secundárias

Esse conjunto de modificações é desencadeado e regulado por um complexo mecanismo neuroendócrino, ainda não completamente esclarecido, e influenciado por fatores genéticos e ambientais. Nota-se a influência de fatores hereditários nos eventos puberais, sobretudo no tocante à variabilidade de tais fenômenos e à sua magnitude, a exemplo das características de pilosidade, tamanho das mamas e idade de ocorrência da primeira menstruação (menarca) $)^{4,5}$. Sob condições ambientais favoráveis, grande parte das variações do crescimento físico na adolescência será ditada predominantemente por fatores genéticos ${ }^{6}$.

Os eventos puberais no sexo feminino iniciam-se mais precocemente quando comparados 
ao sexo masculino, cerca de um a dois anos antes. A primeira manifestação puberal nas meninas é o desenvolvimento do broto ou botão mamário, fenômeno denominado de telarca. No sexo masculino, o início clínico da puberdade é marcado pelo aumento do volume testicular, ao atingir quatro centímetros cúbicos (mililitros), o que é raramente percebido pelo próprio adolescente ${ }^{6,8}$. Na prática clínica, a medida do volume testicular e o acompanhamento de seu desenvolvimento são realizados com auxílio de um orquidômetro (Figura 2).

O desenvolvimento gonadal é marcante nesse período e culmina com o desenvolvimento da capacidade reprodutiva completa do adolescente ${ }^{4}$.

A sequência do desenvolvimento das características sexuais secundárias no adolescente foi sistematizada por Tanner, em 1962 (Tabela 1) $)^{5,11}$. Esse autor descreveu estágios de maturação sexual que são classificados à inspeção durante o exame físico, e variam do Estágio 1 (infantil) ao 5 (adulto), considerando-se o desenvolvimento mamário (M) e a pilosidade pubiana $(P)$ para o sexo feminino, e o desenvolvimento da genitália externa (G) e da pilosidade pubiana $(P)$ para o sexo masculino. A aplicação das pranchas (modelos gráficos) e

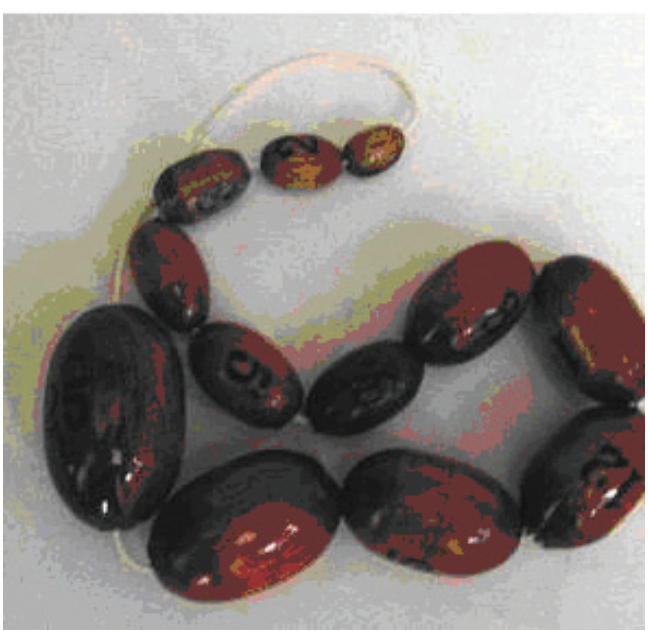

FIGURA 2: A medida testicular deve ser realizada com o orquidômetro, sendo o de Prader observado nessa foto.

da classificação de Tanner faz parte da rotina de avaliação clínica do adolescente, possibilitando a identificação do estágio de maturação sexual em que ele se encontra e sua correlação com outros eventos da puberdade.

\section{Estágios puberais de Tanner}

\section{TABELA 1: Estadiamento maturacional de Tanner ${ }^{11}$}

\section{Desenvolvimento mamário - sexo feminino}

M1 - Mama infantil, com elevação somente da papila.

M2 - Broto mamário. Forma-se uma saliência pela elevação da aréola e da papila. O diâmetro da aréola aumenta e há modificação na sua textura. Há pequeno desenvolvimento glandular subareolar.

M3 - Maior aumento da mama e da aréola, sem separação dos seus contornos. O tecido mamário extrapola os limites da aréola.

M4 - Maior crescimento da mama e da aréola, sendo que esta forma uma segunda saliência acima do contorno da mama (duplo contorno).

M5 - Mama de aspecto adulto, em que o contorno areolar novamente é incorporado ao contorno da mama.

\section{Desenvolvimento genital - sexo masculino}

G1 - Testículos, escroto e pênis de tamanho e proporções infantis.

G2 - Aumento inicial do volume testicular (3-4 ml). Pele do escroto muda de textura e torna-se avermelhada. Aumento do pênis pequeno ou ausente.

G3 - Crescimento do pênis em comprimento. Maior aumento dos testículos e do escroto.

G4 - Aumento do pênis, principalmente em diâmetro e desenvolvimento da glande. Maior crescimento de testículos e escroto, cuja pele torna-se mais enrugada e pigmentada.

G5 - Desenvolvimento completo da genitália, que assume características adultas.

\section{Pilosidade pubiana - sexos feminino e masculino}

P1 - Ausência de pelos pubianos. Pode haver uma leve penugem, semelhante à observada na parede abdominal.

P2 - Aparecimento de pelos longos e finos, levemente pigmentados, lisos ou pouco encaracolados, ao longo dos grandes lábios e na base do pênis.

P3 - Maior quantidade de pelos, agora mais grossos, escuros e encaracolados, espalhando-se esparsamente na região pubiana.

P4 - Pelos do tipo adulto, cobrindo mais densamente a região pubiana, mas sem atingir a face interna das coxas.

P5 - Pilosidade pubiana igual à do adulto, em quantidade e distribuição, invadindo a face interna da coxa.

Obs. Algumas pessoas apresentam extensão dos pelos pela linha alba, acima da região pubiana, constituindo-se o estágio P6. 
Lourenço B, Queiroz LB. Crescimento de desenvolvimento puberal na adolescência.

\section{Correlação entre maturação sexual e estirão}

As diferentes fases do estirão de crescimento se relacionam de maneira peculiar com outros fenômenos da puberdade, como a maturação sexual, de maneira que o estadiamento puberal permite a estimativa do momento de crescimento esquelético do adolescente 6,8 .

No sexo feminino, o início da puberdade (M2) coincide com o início do estirão puberal em sua fase de aceleração, atingindo o máximo da velocidade de crescimento em M3 e desacelerando em M4, momento em que geralmente ocorre a menarca ${ }^{4,6}$. No sexo masculino, o início da puberdade (G2) ocorre ainda em um momento de velocidade de crescimento estável ou pré-puberal. A aceleração do crescimento geralmente ocorre apenas no estágio de G3, e o pico de velocidade de crescimento, em G4, quando se inicia a desaceleração do crescimento6,8. Essa diferença na característica do estirão puberal entre o sexo masculino e o feminino, justifica, em parte, a estatura final do homem ser maior que a da mulher, uma vez que eles permanecem mais tempo na fase de crescimento pré-puberal. Outro aspecto que justifica a diferença de estatura entre os sexos é a magnitude da velocidade de crescimento, ocorrendo um pico de 10 a $12 \mathrm{~cm} /$ ano no sexo masculino, comparado aos 8 a $10 \mathrm{~cm} / a n o$ no sexo feminino.

\section{Marcos puberais de referência}

As idades em que acontecem os eventos puberais apresentam ampla variação individual. Os valores de referência para a população brasileira foram extraídos de um estudo transversal realizado por Colli et al., em Santo André (1978). A telarca ocorre em média aos 9,7 anos $( \pm 3 \text { anos })^{4}$. 0 desenvolvimento mamário pode ser assimétrico, sendo frequente o aparecimento unilateral, seguido algum tempo depois pelo broto contralateral.

A menarca é um evento tardio no desenvolvimento puberal. Tendo importante papel na avaliação do crescimento e desenvolvimento infantopuberal, a observação das características sexuais secundárias e a idade da menarca são recomendadas no acompanhamento periódico do crescimento da criança. Sendo assim, além de seu contexto biológico, social e psíquico, a menarca tem relevante importância como marco do crescimento e desenvolvimento humano, e é o indicador de maturidade sexual mais usado. A variabilidade da data da primeira menstruação envolve características genéticas e influência ambiental, com interações complexas. A menarca ocorre cerca de dois anos após o início da puberdade (M2), geralmente no estágio M4 de Tanner, portanto, em época de desaceleração do crescimento. $O$ crescimento é limitado em média a 4-6 cm nos dois-três anos pós-menarca ${ }^{3}$. A idade média de ocorrência da menarca é de 12,2 anos $( \pm 2,4 \text { anos })^{3,4}$. Os ciclos iniciais da adolescente podem apresentar certa irregularidade nos primeiros dois a quatro anos (ciclos anovulatórios).

A idade média do início do aumento testicular é de 10,9 anos ${ }^{4}$. Nota-se que esse aumento precede o aumento peniano, motivo clássico de preocupação comum entre os meninos. A ejaculação também é um evento tardio no desenvolvimento puberal masculino e, pode se manifestar inicialmente com emissões noturnas involuntárias ${ }^{8}$.

O crescimento dos pelos axilares e faciais se segue ao dos pelos pubianos. A mudança vocal, decorrente do aumento da laringe por ação androgênica, ocorre tardiamente no processo puberal masculino.

A ginecomastia é também um evento puberal comum nos meninos. Conceitua-se como o aumento glandular da mama masculina que, clinicamente, é caracterizada na palpação por um disco de consistência firme, subareolar e móvel, não aderente à pele ou ao tecido subjacente. Deve ser diferenciada da lipomastia ou adipomastia, presente em meninos pré-púberes ou púberes obesos, que é o aumento mamário devido exclusivamente ao aumento do tecido gorduroso; esse geralmente é bilateral e de consistência amolecida à palpação. A ginecomastia tem como substrato etiológico provável a existência de um desequilíbrio na relação hormonal, na qual os androgênios (ação inibidora do crescimento mamário) estão em proporção menor que os estrogênios, que estimulam o tecido mamário. A ginecomastia puberal tem pico de incidência por volta dos 13 aos 14 anos, coincidindo com o estágio maturacional G3 de Tanner; desaparece espontaneamente em menos de um ano na metade dos casos, dentro de dois anos em $75 \%$, e dentro de três anos em $90 \%$ dos pacientes ${ }^{6}$.

\section{CONCLUSÃO}

O conhecimento dos fenômenos puberais normais constitui-se como um dos alicerces básicos da atenção à saúde dos adolescentes. As queixas, dúvidas e preocupações relacionadas ao crescimento e desenvolvimento puberal são comuns na prática clínica, e é obrigação de qualquer médico que se proponha a atender adolescentes e jovens a orientação técnica adequada em relação a essa demanda. 
Lourenço B, Queiroz LB. Growth and puberal development in adolescence. Rev Med (São Paulo). 2010 abr.-jun.;89(2):70-5.

ABSTRACT: Puberty is a period of adolescence characterized by the biological changes that occur during this period of life. Lasting from two to four years, puberty is marked by linear skeletal growth; changes in body shape and composition; organ and system development and development of gonads and secondary sexual characters. This article aims to discuss these events, as well as the factors that might influence them.

KEY WORDS: Adolescent; Growth and development; Puberty.

\section{REFERÊNCIAS}

1.Barnes HV. Physical growth and development during puberty. Med Clin North Am. 1975;59:1305-17.

2.Behrman RE, Vaughan VC. Nelson essentials of pediatrics. Philadelphia: WB Saunders; 1990. p.151-77.

3. Castilho SD, Barros Filho AA. Crescimento pós-menarca. Arq Bras Endocrinol Metab. 2000;44:195-204.

4.Colli AS. Maturação sexual na população brasileira: limites de idade. J Pediatr. 1986;60:173-5.

5. Marshall WA, Tanner SM. Variations in pattern of puberal chances in girls. Arch Dis Child. 1969;44:291-303.

6. Neinstein LS. Adolescent health care: a practical guide. In: Neinstein LS, Kaufman FR. Abnormal growth and development. Philadelphia: Lippincott Williams and Wilkins; 2002.

7.Saito MI. A avaliação nutricional na adolescência: a escolha do referencial. J Pediatria. 1993;69:165-75.
8. Saito MI, Silva LEV, Leal MM. Adolescência: prevenção e risco. 2a ed. São Paulo: Atheneu; 2008.

9. Sedenho N, Freitas JA. Fatores que influenciam a ocorrência da menarca. J Bras Ginecol. 1984;94:303-8.

10. Tanner JN. Age of menarche in a history of the study of human growth. Cambridge: University Press; 1981.

11. Tanner JN. Growth at adolescence with a general consideration of the effects of hereditary and environmental factors upon growth and maturation from birth to maturity. 2nd ed. Oxford: Blackwell Scientific Publications; 1962.

12. Vitalle MSS, Tomioka CY, Juliano $Y$, Amancio OMS. Índice de massa corporal, desenvolvimento puberal e sua relação com a menarca. Rev Assoc Med Bras. 2003;49(4):429-33.

Artigo recebido em: 10/06/2010.

Artigo aceito em: 10/06/2010. 